\title{
Systematic review of the effects of fatigue on spatiotemporal gait parameters
}

\author{
Fabio Augusto Barbieri ${ }^{\mathrm{a}, \mathrm{b}, *}$, Paulo Cezar Rocha dos Santos ${ }^{\mathrm{a}}$, Ellen Lirani-Silva ${ }^{\mathrm{a}}$, Rodrigo Vitório ${ }^{\mathrm{a}}$, \\ Lilian Teresa Bucken Gobbi ${ }^{\mathrm{a}}$ and Jaap H. van Diëen ${ }^{\mathrm{b}}$ \\ ${ }^{a}$ UNESP, São Paulo State University at Rio Claro, LEPLO, São Paulo, Brazil \\ ${ }^{\mathrm{b}}$ Research Institute MOVE, Faculty of Human Movement Sciences, VU University Amsterdam, Amsterdam, The \\ Netherlands
}

\begin{abstract}
.
PURPOSE: This systematic review aimed to assess changes on spatiotemporal gait parameters due to fatigue.

MATERIAL AND METHOD: A search was carried out in literature published from 1950 to December 2010 and retrieved 771 articles using terms referring to walking and fatigue in the title, abstract or keywords. Two researchers assessed the selection and quality of each article independently.

RESULTS: Seven studies were selected for this systematic review, two of which reported on the same data set. Several spatiotemporal parameters were reported to change with fatigue, but the few variables studied in multiple studies, gait speed and stride or step length and stride time, were affected only in single studies. Fatigue appears to modulate spatiotemporal gait parameters, but the effects of fatigue appear to be dependent on the muscles that were fatigued, and age that appears to be a moderator of the effects of fatigue on gait.
\end{abstract}

Keywords: Gait, muscle fatigue, spatiotemporal parameters, motor control

\section{Introduction}

Fatigue is used to describe a number of phenomena with a variety of causes [1], but may be more strictly defined as any exercise-induced reduction in the ability of a muscle to generate force or power [2], or failure to continue working at a given exercise intensity [3]. Fatigue development is mediated by a range of individual factors such as the presence of pathology, age (older adults fatigue faster than young adults), physical activity level (inactive individuals fatigue faster than active individuals), and the type of activity performed. Fatigue not only affects elderly and diseased popula-

* Address for correspondence: Prof. Fabio Augusto Barbieri, Universidade Estadual Paulista, UNESP, IB, Rio Claro, Laboratório de Estudos da Postura e da Locomoção, Departamento de Educação Física, Avenida 24-A, 1515, Bela Vista, CEP: 13.506-900-Rio Claro, São Paulo, Brasil. Tel.: +55 193526 4320; Fax: +55 193534 6436; E-mail: barbieri_rc@hotmail.com. tions, as $5-56 \%$ of employees in 15 European countries reported fatigue at work [4] and one-third of the US workforce experienced fatigue in the workplace [5].

Fatigue is considered as a safety mechanism to prevent injury $[6,7]$, but it may have negative effects on activities of daily living (ADL) [8]. For example, walking distance, cleaning the house, general household chores and getting exercise are limited by fatigue [9]. Therefore, fatigue can contribute to disability, regarding occupational activities and ADL and as such be the cause of a deterioration in independence and quality of life. Furthermore fatigue adversely affects proprioception, movement coordination and reaction times [10], and consequently has negative effects on motor control [11]. For example, fatigue may compromise balance control and predispose to falls [10,12-15]. In an investigation of slip and fall accidents among postal delivery workers in the UK [16], fatigue was found to be a precursor of $25 \%$ of all fall incidents. 
The literature provides some evidence of fatiguerelated changes of the biomechanical characteristics of gait, indicating that a new segmental organization occurs in the presence of muscle fatigue, as observed by changes of spatiotemporal gait parameters $[10,13-15$, 17-21]. However, a comprehensive overview of which gait parameters and how these parameters are changed by fatigue is lacking. Therefore, a systematic review was performed with the aim to assess changes of spatiotemporal gait parameters due to fatigue. An understanding of fatigue effects on walking could help to avoid falls, slip, trips and injuries [17], and as such improve quality of life.

\section{Methods}

\subsection{Search strategy}

The initial search strategy was performed to identify all articles on the effect of fatigue on spatiotemporal gait parameters. An electronic search was conducted in 6 databases: Cochrane Library, Embase, Medline ISI, PsycInfo, PubMed, and Web of Science - ISI. We searched articles from 1950 to December 2010. Targeted searching of frequently cited journals, authors and article reference lists ensured that all relevant articles were located. To be deemed relevant for review, an article had to contain terms referring to walking or gait and fatigue in the title, abstract or keywords. Key terms within the search strategy were also matched using medical subject headings (MeSH) in databases such as Medline. Articles on animal studies, robotics, or children and reviews, methodological or descriptive papers were excluded using another set of key terms. As an example, the following search strategy was used in the PubMed: "gait OR walking AND fatigue" searched in the title/abstract, with following limits: language - English; type of article - clinical trial, randomized controlled trial and journal article; species: humans.

\subsection{Section criteria and quality assessment}

The initial yield was obtained by combining all original articles from electronic databases and targeted searches. Articles not relating to walking were excluded during an initial screening of titles and abstracts. The remaining abstracts were checked against the inclusion/exclusion criteria. The full text was examined if the abstract contained insufficient information to decide on inclusion.
Two reviewers screened all articles for inclusion. A set of guidelines and examples for completing article reviews was provided to both reviewers to improve inter-reviewer reliability. The two researchers independently assessed the selection and quality of each article. When there were discrepancies between the completed quality appraisal and data extraction forms of the two reviewers, a third reviewer was consulted to solve the divergence. Only English language articles were considered for analysis. Studies that investigated effects of fatigue on gait in healthy young, middleaged and older adults, but not pathological individuals were initially selected. If a study investigated walking freely on flat ground and another form of locomotion or walking with a concurrent task, only details pertaining to effects of fatigue in the walking freely on flat ground condition were considered. Articles were included in the systematic review if they investigated the effects of fatigue in walking freely on flat ground on spatiotemporal parameters. As differences in terminology and writing style exist between researchers, a list of synonyms for spatiotemporal (step or stride length and width, stride time, speed, velocity, acceleration, spatiotemporal and spatial-temporal) was used for each key term to identify all relevant articles. Studies on treadmill walking were excluded, because gait adaptations on a treadmill may be different from those in over ground walking [22].

The quality of a study was defined as its capacity to avoid potential bias and to generate results that can be generalized. This definition encompasses both internal and external validity as dimensions of quality. Internal validity refers to the accuracy of the measurements. External validity refers to whether the results can be generalized to the population of interest. We used a quality appraisal tool developed by Galna et al. [23], which is specific for this type of systematic review. A scoring system was used to quantify the quality of each study and to assess its methodological strengths and weaknesses. Each question on the quality appraisal tool was scored with a maximum of one, indicating high quality research, with a 0.5 score indicating lack of detail or unclear information and a zero score indicating low quality. A study was considered of good quality when the summed quality score was higher than seven. A similar quality score was used previously [24]. 
Table 1

Methodological quality appraisal results. Y - yes; YL - yes, lacking detail or clarity; N-No; AV - average. A score of one indicated high quality research, 0.5 indicated lack of detail or unclear information, and zero indicated lower quality

\begin{tabular}{|c|c|c|c|c|c|c|c|}
\hline Question & Scoring criteria & $\begin{array}{l}\text { Yoshino } \\
\text { et al. [18] }\end{array}$ & $\begin{array}{l}\text { Kavanagh } \\
\text { et al. [20] }\end{array}$ & $\begin{array}{l}\text { Helbostad } \\
\text { et al. [15] }\end{array}$ & $\begin{array}{c}\text { Parijat et al. } \\
{[13,14]}\end{array}$ & $\begin{array}{l}\text { Granacher } \\
\text { et al. [21] }\end{array}$ & $\begin{array}{c}\text { Olson } \\
{[19]}\end{array}$ \\
\hline 1. Research aims or questions stated clearly & $1-\mathrm{Y} ; 0.5-\mathrm{YL} ; 0-\mathrm{N}$ & 1 & 1 & 1 & 1 & 1 & 1 \\
\hline 2. Participants detailed & $\begin{array}{l}\text { Number } \\
\text { Age } \\
\text { Sex } \\
\text { Height } \\
\text { Sub total }\end{array}$ & $\begin{array}{c}1 \\
1 \\
1 \\
0 \\
0.75\end{array}$ & $\begin{array}{l}1 \\
1 \\
1 \\
1 \\
1\end{array}$ & $\begin{array}{c}1 \\
1 \\
1 \\
0 \\
0.75\end{array}$ & $\begin{array}{l}1 \\
1 \\
1 \\
1 \\
1\end{array}$ & $\begin{array}{l}1 \\
1 \\
1 \\
1 \\
1\end{array}$ & $\begin{array}{l}1 \\
1 \\
1 \\
1 \\
1\end{array}$ \\
\hline 3. Recruitment and sampling methods described & $1-\mathrm{Y} ; 0.5-\mathrm{YL} ; 0-\mathrm{N}$ & 0 & 0.5 & 0.5 & 0 & 0.5 & 0 \\
\hline 4. Inclusion and exclusion criteria detailed & $1-\mathrm{Y} ; 0.5-\mathrm{YL} ; 0-\mathrm{N}$ & 0 & 0.5 & 1 & 0.5 & 1 & 0.5 \\
\hline 5. Controlled covariates & $\begin{array}{l}\text { Height } \\
\text { Walking speed } \\
\text { Age } \\
\text { Gender } \\
\text { Limb asymmetries } \\
\text { Strength } \\
\text { Sub total }\end{array}$ & $\begin{array}{r}0 \\
1 \\
1 \\
1 \\
0 \\
0 \\
0.5\end{array}$ & $\begin{array}{c}0 \\
0 \\
1 \\
1 \\
0 \\
1 \\
0.5\end{array}$ & $\begin{array}{c}0 \\
1 \\
1 \\
0 \\
0 \\
0 \\
0.33\end{array}$ & $\begin{array}{c}0 \\
0 \\
1 \\
1 \\
0 \\
1 \\
0.5\end{array}$ & $\begin{array}{c}0 \\
1 \\
1 \\
1 \\
1 \\
1 \\
0.83\end{array}$ & $\begin{array}{c}0 \\
1 \\
1 \\
1 \\
0 \\
0 \\
0.5\end{array}$ \\
\hline 6. Key outcome variables clearly described & $1-\mathrm{Y} ; 0.5-\mathrm{YL} ; 0-\mathrm{N}$ & 1 & 1 & 1 & 1 & 1 & 1 \\
\hline 7. Adequate methodology able to repeat study & $\begin{array}{l}\text { Participant sampling } \\
\text { Equipment } \\
\text { Procedure } \\
\text { Data processing } \\
\text { Statistical analysis } \\
\text { Sub total }\end{array}$ & $\begin{array}{r}0 \\
1 \\
1 \\
1 \\
1 \\
0.8\end{array}$ & $\begin{array}{l}1 \\
1 \\
1 \\
1 \\
1 \\
1\end{array}$ & $\begin{array}{l}1 \\
1 \\
1 \\
1 \\
1 \\
1\end{array}$ & $\begin{array}{l}1 \\
1 \\
1 \\
1 \\
1 \\
1\end{array}$ & $\begin{array}{l}1 \\
1 \\
1 \\
1 \\
1 \\
1\end{array}$ & $\begin{array}{l}1 \\
1 \\
1 \\
1 \\
1 \\
1\end{array}$ \\
\hline 8. Methodology able to answer research question & $\begin{array}{l}\text { Participant sampling } \\
\text { Equipment } \\
\text { Procedure } \\
\text { Data processing } \\
\text { Statistical analysis } \\
\text { Sub total }\end{array}$ & $\begin{array}{l}1 \\
1 \\
1 \\
1 \\
1 \\
1\end{array}$ & $\begin{array}{l}1 \\
1 \\
1 \\
1 \\
1 \\
1\end{array}$ & $\begin{array}{l}1 \\
1 \\
1 \\
1 \\
1 \\
1\end{array}$ & $\begin{array}{l}1 \\
1 \\
1 \\
1 \\
1 \\
1\end{array}$ & $\begin{array}{l}1 \\
1 \\
1 \\
1 \\
1 \\
1\end{array}$ & $\begin{array}{l}1 \\
1 \\
1 \\
1 \\
1 \\
1\end{array}$ \\
\hline 9. Reliability of the methodology stated & $1-\mathrm{Y} ; 0-\mathrm{N}$ & 0 & 0 & 0 & 0 & 0 & 0 \\
\hline 10. Internal validity of the methodology stated & $1-\mathrm{Y} ; 0-\mathrm{N}$ & 0 & 0 & 0 & 0 & 0 & 0 \\
\hline $\begin{array}{l}\text { 11. Research questions answered adequately } \\
\text { in the discussion }\end{array}$ & $1-\mathrm{Y} ; 0-\mathrm{N}$ & 1 & 1 & 1 & 1 & 1 & 1 \\
\hline 12. Key findings supported by the results & $1-\mathrm{Y} ; 0-\mathrm{N}$ & 1 & 1 & 1 & 1 & 1 & 1 \\
\hline $\begin{array}{l}\text { 13. Key findings interpreted in a logical manner } \\
\text { which is supported by references }\end{array}$ & $1-\mathrm{Y} ; 0-\mathrm{N}$ & 1 & 1 & 1 & 1 & 1 & 1 \\
\hline 14. Clinical implications stated & $1-\mathrm{Y} ; 0.5-\mathrm{YL} ; 0-\mathrm{N}$ & 0.5 & 1 & 1 & 1 & 1 & 1 \\
\hline
\end{tabular}

\section{Results}

\subsection{Yield and quality assessment}

The search in the databases revealed 771 articles (Fig. 1). Following abstract review, 81 articles were related to the effect of fatigue on spatiotemporal parameters of gait. The final yield included seven articles that analyzed effects of fatigue on spatiotemporal parameters of gait. Two papers reported data collected in the same experiment $[13,14]$, leaving six papers for review. Table 1 summarizes the quality assessment for these papers. Overall, quality was satisfactory since all papers obtained a quality score higher than 8 . The arti- cles stated the aims with sufficient clarity, gave an appropriate description of the participants, detailed the key outcomes clearly, employed a suitable methodology, discussed the results appropriately and indicated implications. One important shortcoming in the statistical analysis was noted for the study by Yoshino et al. [18]. In this study, subjects were sub-grouped based on the changes in spatiotemporal parameters that occurred due to fatigue (duration of stride time). This makes it impossible to judge whether changes in spatiotemporal parameters were significant at the group level. Nevertheless the results of the largest subgroup (8 vs 4 subjects) were included in the review. Most investigations controlled for age and gender, but typically did not control for height, limb asymmetries and 
Table 2

Description articles that examined fatigue in walking. YA - young adults; OA - older adults; MVC - maximal voluntary contraction; ST - stride time; SL - stride length; SPL - step length; GS - gait speed; SPW - step width; HCV - heel contact velocity; TA - transitional acceleration

\begin{tabular}{|c|c|c|c|c|c|}
\hline Authors & Objectives & Group & Fatigue protocol & Gait parameters & $\begin{array}{l}\text { Fatigue } \\
\text { effects* }\end{array}$ \\
\hline $\begin{array}{l}\text { Yoshino } \\
\text { et al. [18] }\end{array}$ & $\begin{array}{l}\text { To examine whether gait } \\
\text { patterns and physiological } \\
\text { rhythms are affected by fa- } \\
\text { tigue }\end{array}$ & $\begin{array}{l}12 \text { males: Group } \mathrm{A}-23.1 \pm \\
1.5 \mathrm{yrs} \text { (long gait cycle time in } \\
\text { the } 2^{\text {nd }} \text { half of protocol) and } \\
\text { Group B }-20.7 \pm 1.2 \mathrm{yrs} \text { (short } \\
\text { gait cycle time) }\end{array}$ & $\begin{array}{l}\text { To walk } 3 \mathrm{~h} \text { continuously } \\
\text { at preferred pace on ground } \\
\text { level }\end{array}$ & $\begin{array}{l}\text { ST, variability of ST, } \\
\text { trunk acceleration }\end{array}$ & YES** \\
\hline $\begin{array}{l}\text { Kavanagh } \\
\text { et al. [20] }\end{array}$ & $\begin{array}{l}\text { To examine how fatigue af- } \\
\text { fects the ability to maintain } \\
\text { head stability during walk- } \\
\text { ing }\end{array}$ & 8 males $(23 \pm 4$ yrs $)$ & $\begin{array}{l}\text { Isometric contraction of } \\
\text { lumbar and cervical erector } \\
\text { spinal muscles: } 60 \% \text { MVC } \\
\text { (30 s contraction, } 30 \text { s rest) }\end{array}$ & GS, ST, SPL, cadence & NO \\
\hline $\begin{array}{l}\text { Helbostad } \\
\text { et al. [15] }\end{array}$ & $\begin{array}{l}\text { To investigate the effect } \\
\text { of fatigue on trunk and foot } \\
\text { level gait characteristics }\end{array}$ & $\begin{array}{l}17 \text { females and } 10 \text { males (fa- } \\
\text { tigue group: } 78.2 \pm 5 \mathrm{yrs} \text {; con- } \\
\text { trol group: } 80.4 \pm 4.9 \mathrm{yrs} \text { ) }\end{array}$ & $\begin{array}{l}\text { Sit-to-stand repeatedly } \\
\text { from a chair at a fast speed } \\
\text { until felt too exhausted }\end{array}$ & $\begin{array}{l}\text { Trunk acceleration } \\
\text { amplitudes and variability, } \\
\text { SPW, variability of SPL } \\
\text { SPL, GS, variability of SPW }\end{array}$ & NO \\
\hline $\begin{array}{l}\text { Parijat } \\
\text { et al. } \\
{[13,14]}\end{array}$ & $\begin{array}{l}\text { To investigate the effects of } \\
\text { lower muscle fatigue on gait } \\
\text { characteristics }\end{array}$ & $\begin{array}{l}6 \text { females and } 10 \text { males }(24.6 \pm \\
3.5 \mathrm{yrs})\end{array}$ & $\begin{array}{l}\text { Isokinetic knee extension } \\
\text { at } 70 \% \text { MVC until force } \\
\text { drops below } 60 \% \mathrm{MVC}\end{array}$ & $\begin{array}{l}\text { HCV, TA, } \\
\text { GS }\end{array}$ & $\begin{array}{l}\text { YES } \\
\text { NO }\end{array}$ \\
\hline $\begin{array}{l}\text { Granacher } \\
\text { et al. [21] }\end{array}$ & $\begin{array}{l}\text { To examine the effects of fa- } \\
\text { tigue on gait under single } \\
\text { and dual-task conditions }\end{array}$ & $\begin{array}{l}16 \text { females and } 16 \text { males (YA: } \\
24.3 \pm 1.4 \text { yrs; OA: } 71.9 \pm \\
5.5 \mathrm{yrs} \text { ) }\end{array}$ & $\begin{array}{l}\text { Max. isokinetic knee } \\
\text { extension until force drops } \\
\text { below } 50 \% \text { MVC }\end{array}$ & $\begin{array}{l}\text { YA: GS, SL } \\
\text { OA: GS, SL; YA and } \\
\text { OA: SL variability }\end{array}$ & $\begin{array}{l}\text { YES } \\
\text { NO }\end{array}$ \\
\hline $\begin{array}{l}\text { Olson } \\
{[19]}\end{array}$ & $\begin{array}{l}\text { To study changes in muscle } \\
\text { activation and gait parame- } \\
\text { ters with fatigue }\end{array}$ & 14 females $(27.5 \pm 12$ yrs $)$ & $\begin{array}{l}\text { Isometric trunk extension at } \\
50 \% \text { or } 70 \% \text { MVC until force } \\
\text { drops below } 20 \% \text { MVC }\end{array}$ & GS, ST, SL & $\mathrm{NO}$ \\
\hline
\end{tabular}

${ }^{*}$ We indicated only statistical effects of fatigue for free gait. ${ }^{* *}$ In largest sub-group defined post-hoc based on increase in average stride time.

Table 3

Description of articles' results per parameter. $\uparrow$ - increase after fatigue; $\downarrow$-decrease after fatigue; $\sim-$ similar after fatigue; YA - young adults; $\mathrm{OA}$ - older adults

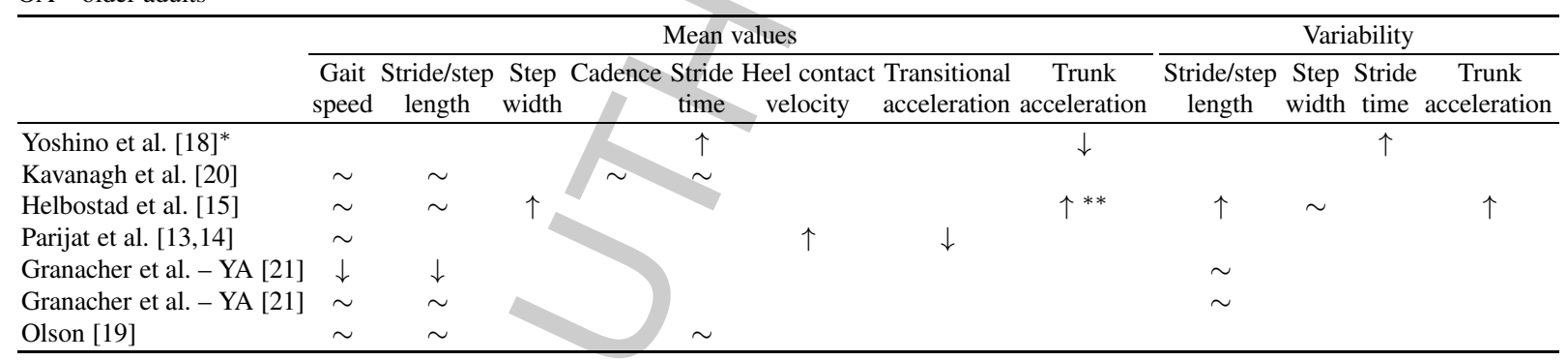

${ }^{*}$ In largest sub-group defined post-hoc based on increase in average stride time. ${ }^{* *}$ In frail elderly only.

strength differences between groups. Details provided were adequate to replicate the studies, although inclusion/exclusion criteria were often not reported. No study completely described the recruitment and sampling methods. The reliability of key outcome measures and the internal validity of the key outcome measures were reported in only one study.

\subsection{Sample characteristics}

Both male and female participants were recruited in the studies analyzed (Table 2), except for three stud- ies in which participants of one sex were recruited: one study included only females [19] and two studies included only males $[18,20]$. Four studies $[14,18-20]$ analyzed the influence of fatigue in young adults, while one paper [15] evaluated the effect of fatigue on gait in elderly. A single study [21] compared the effects of fatigue on gait between age groups (young vs older adults). In the seven papers, the mean age of the young adults ranged from 20 to 34 years and of the older adults it ranged from 71 to 81 years. 


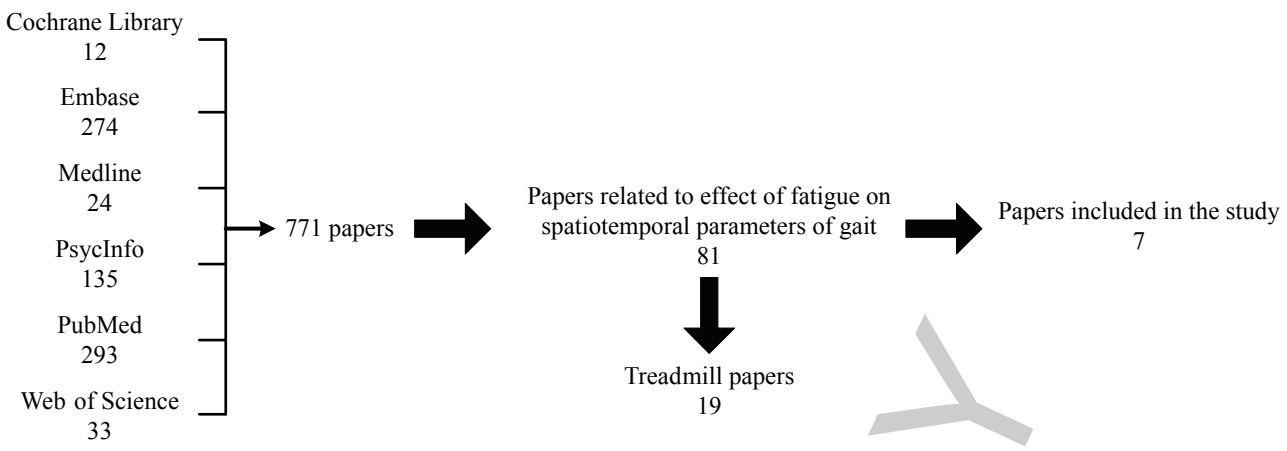

Fig. 1. Flowchart of articles included for review. The number under the text indicates the number of original articles (i.e., not duplicates) at each stage of the search.

\subsection{Methods to induce fatigue}

Protocols used to induce fatigue differed between the studies analyzed including isometric contractions at different percentages of maximal voluntary contraction $[19,20]$, isokinetic exercise $[13,14,21]$, repeated sit-to-stand transfers [15] and walking itself [18]. When isometric and isokinetic contraction were used, the loads ranged from $50 \%$ to $70 \%$ of the maximum. Regarding the time to execute the fatigue protocol, studies used a fixed time period [18,20], or the time that the participant could maintain performance at a given contraction level $[13,14,19,21]$, or the time until the participant felt too exhausted to do more repetitions [15]. Moreover, different muscles were fatigued. In three studies the knee extensor muscles were fatigued [13-15,21], in two studies the trunk muscles $[19,20]$, and in one study the lower limb muscles [18]. Fatigue as defined by a decrease in force producing capacity of the muscles exercised was confirmed to be present at the end of or after the fatigue protocol in all studies but two [15,18]. The latter studies did report subjective indications of fatigue and Yoshino et al. [18] did show indications of muscle fatigue in the EMG of the tibialis anterior muscle in the largest sub-group of subjects in their study.

\subsection{Spatiotemporal parameters}

The spatiotemporal parameters analyzed in the included studies were gait speed, stride and step length, stride time, step width, cadence, trunk accelerations, transitional acceleration and heel contact velocity. In addition, some studies addressed the variability of selected spatiotemporal parameters: stride time, stride length, step width and trunk accelerations. The results are summarized per study in Table 2 and per parame- ter studied in Table 3. Young adults reduced, or maintained gait speed and stride/step length, and increased stride time and its variability, and heel contact velocity with fatigue. In healthy elderly, the main changes found were increased step width and stride length variability that with fatigue.

\section{Discussion}

The following question was addressed in this systematic review: What are the effects of fatigue on the spatiotemporal gait parameters? The literature retrieved was limited in numbers and used widely different protocols to induce fatigue and a range of different dependent variables. Strikingly the most commonly studied variables, gait speed, step or stride length and stride time did not appear to be affected in a majority of studies. Nevertheless, several other gait parameters appear to change due to fatigue, although many were reported in only one or two studies.

The effects of fatigue on the spatiotemporal gait parameters appear dependent on the muscles that were fatigued, with changes being more evident when leg muscles were fatigued than when postural (trunk) muscles were fatigued. However, the studies addressing trunk muscle fatigue did not report variables related to trunk movement (e.g., medial-lateral trunk acceleration or angular displacement). Thus, when trunk muscles are fatigued, individuals appear not to modulate lower limb gait kinematics. The knee extensor muscles, which obviously have more specific roles in gait, controlling balance [25] and positioning of the limb [26], were fatigued in most of the studies, and although lower limb kinematics appear to be affected the diversity of dependent variables studied precludes definitive conclusions. 
As for exercise type, the diversity of fatigue protocols used does not allow definitive conclusions. Although isometric protocols may offer a safe, selective and standardized way of fatiguing muscles [7], the strategy of neuromuscular recruitment varies according to the task (isometric or isotonic task) [19] and hence results may be influenced by the task used [1] Therefore, inducing fatigue under dynamic conditions may be more relevant to physical performance during daily living activities [27]. Induction of fatigue through a functional task like walking or repeated sit-to-stand transfers as used in some of the studies likely has the advantage that results are more representative for daily life conditions. In addition to the type of exercise, the duration of the fatigue protocol may affect the results, specifically because the fatigue effects are measured over a period of walking after the protocol during which recovery may occur. Recovery after short, highintensity exercise may be relatively fast [28]. To reflect daily activity, it has been recommended that the duration of the exercise should be controlled rather than the task intensity or workload [1].

Only one study [21] directly compared young and old adults, hence no definitive conclusion on the moderating effect of age can be made. The results of this study [21], with reductions in gait speed and stride length in young adults only, suggest that young adults use a more conservative strategy $[29,30]$ to deal with fatigue. Also other studies with young adult participants only showed indications of a more conservative strategy, although heel contact velocity increased with fatigue, which would increase slip risk [31]. Overall, young adults seem to modulate gait performance to increase safety. Studies on older adults only showed indications that their gait pattern is not robust against fatigue, such as increased trunk accelerations and increased variability of stride length and trunk acceleration, possibly as direct effects of fatigue and concomitant loss of control [32,33]. As possible adaptations, older adults did show an increase of step width [15, 25], which would increase medio-lateral stability of gait [34]. Remarkably, no evidence was found for reductions in gait speed and stride length in older adults. An increase in gait speed may decrease the mediolateral displacement of the center of mass, further contributing to medio-lateral balance control [35-37]. There may be a U-shaped relation between fall risk and gait speed [25]. Possibly young adults normally walk above the speed at which their fall risk is minimal, making a decrease in speed useful for them to decrease their risk in case of fatigue. A similar decrease might not be effective in older adults who already prefer lower walking speeds than young adults [31].

With fatigue spatiotemporal gait parameters appear to be modulated. However, the adjustments appear to be dependent on the muscles that were fatigued, the type and duration of exercise and possibly the subjects' age. Fatigue coincides with a decrease in muscle strength $[1,38]$, reduced proprioceptive acuity, and delayed neuromuscular responses [39]. These changes are likely to increase fall risk during gait. Some of the changes in spatiotemporal gait parameters (increased heel contact velocity, increased trunk acceleration and increased variability of stride length) appear to reflect this increased risk of falling. However, simultaneously, others changes (increased step width, reduced gait speed and stride length) may reflect adaptations to counteract the increase of the risk of falling.

Several limitations of this review are evident. Despite the importance of fatigue in daily activities and gait, few studies have addressed this issue. More studies are required to understand the effects of fatigue on gait, with careful consideration of subjects' age and the muscles involved as well as the type of exercise done to cause fatigue. From the literature reviewed, we conclude that: a) fatigue coincides with changes in the spatiotemporal parameters of gait; $b$ ) the effects of fatigue on the spatiotemporal gait parameters appear dependent on the muscles that are fatigued; c) age may be a moderator of the effects of fatigue on gait.

\section{Acknowledgments}

The authors thank CAPES.

\section{References}

[1] Egerton T, Brauer SG, Cresswell AG. Fatigue after physical activity in healthy and balance-impaired elderly. J Aging Phys Act. 2009; 17: 89-105.

[2] Edwards, RHT. Human muscle function and fatigue. Ciba Foundation Symposium. 1981; 82: 1-18.

[3] Booth FW, Thomason DB. Molecular and cellular adaptation of muscle in response to exercise: Perspectives of various models. Physiol Rev. 1991; 71: 541-585.

[4] Benavides FG, Benach J, Ez-Roux AV, Roman C. How do types of employment relate to health indicators? Findings from the Second European Survey on Working Conditions. Br Med J. 2000; 54: 494-501.

[5] Swaen GM, Van Amelsvoort LG, Bultmann U, Kant IJ. Fatigue as a risk factor for being injured in an occupational accident: Results from the maastricht cohort study. Occup Environ Med. 2003; 60(1): i88-92. 
[6] Abbiss CR, Laursen PB. Models to explain fatigue during prolonged endurance cycling. Sports Med. 2005; 35: 865-898.

[7] Ratel S, Duche P, Williams CA. Muscle fatigue during highintensity exercise in children. Sports Med. 2006; 36: 10311065.

[8] Liao S, Ferrell BA. Fatigue in an older population. J Am Geriatr Soc. 2000; 48: 426-430.

[9] Tralongo P, Respini D, Ferrau F. Fatigue and aging. Crit Rev Oncol Hematol. 2003; 8: S57-64.

[10] Lin D, Nussbaum MA, Seol H, Singh NB, Madigan ML, Wojcik LA. Acute effects of localized muscle fatigue on postural control and patterns of recovery during upright stance: Influence of fatigue location and age. Eur J Appl Physiol. 2009; 106: 425-434.

[11] Ribeiro F, Mota J, Oliveira J. Effect of exercise-induced fatigue on position sense of the knee in the elderly. Eur J Appl Physiol. 2007; 99: 379-385.

[12] Helbostad JL, Sturnieks DL, Menant J, Delbaere K, Lord SR, Pijnappels M. Consequences of lower extremity and trunk muscle fatigue on balance and functional tasks in older people: A systematic literature review. BMC Geriatr. 2010; 10: 56.

[13] Parijat P, Lockhart TE. Effects of lower extremity muscle fatigue on the outcomes of slip-induced falls. Ergonomics. 2008; 51: 1873-1884.

[14] Parijat P, Lockhart TE. Effects of quadriceps fatigue on the biomechanics of gait and slip propensity. Gait Posture. 2008; 28: $568-573$

[15] Helbostad JL, Leirfall S, Moe-Nilssen R, Sletvold O. Physical fatigue affects gait characteristics in older persons. J Gerontol A Biol Sci Med Sci. 2007; 62: 1010-1015.

[16] Bentley TA, Haslam RA. Slip, trip and fall accidents occurring during the delivery of mail. Ergonomics. 1998; 41: 18591872 .

[17] Murdock GH, Hubley-Kozey CL. Effect of a high intensity quadriceps fatigue protocol on knee joint mechanics and muscle activation during gait in young adults. Eur J Appl Physiol. 2012, 112: 439-449.

[18] Yoshino K, Motoshige T, Araki T, Matsuoka K. Effect of prolonged free-walking fatigue on gait and physiological rhythm. J Biomech. 2004; 37: 1271-1280.

[19] Olson MW. Trunk extensor fatigue influences trunk muscle activities during walking gait. J Electromyogr Kinesiol. 2010; 20: $17-24$

[20] Kavanagh JJ, Morrison S, Barrett, RS. Lumbar and cervical erector spinae fatigue elicit compensatory postural responses to assist in maintaining head stability during walking. J Appl Physiol. 2006; 101: 1118-1126.

[21] Granacher U, Wolf I, Wehrle A, Bridenbaugh S, Kressig RW. Effects of muscle fatigue on gait characteristics under single and dual-task conditions in young and older adults. J Neuroeng Rehabil. 2010; 7: 56.

[22] Lee SJ, Hidler J. Biomechanics of overground vs. treadmill walking in healthy individuals. J Appl Physiol. 2008; 104: 747-755.
[23] Galna B, Peters A, Murphy AT, Morris ME. Obstacle crossing deficits in older adults: A systematic review. Gait Posture. 2009; 30: 270-275.

[24] Bragge P, Bialocerkowski A, McMeeken J. A systematic review of prevalence and risk factors associated with playingrelated musculoskeletal disorders in pianists. Occup Med. 2006; 56: 28-38

[25] Helbostad JL, Moe-Nilssen R. The effect of gait speed on lateral balance control during walking in healthy elderly. Gait Posture. 2003; 18: 27-36.

[26] Vila-Chã C, Riis S, Lund D, Moller A, Farina D, Falla D. Effect of unaccustomed eccentric exercise on proprioception of the knee in weight and non-weight bearing tasks. J Electromyogr Kines. 2011; 21: 141-147.

[27] McNeil CJ, Rice CL. Fatigability is increased with age during velocity-dependent contractions of the dorsiflexors. J Gerontol A Biol Sci Med Sci. 2007; 62: 624-629.

[28] Lattier G, Millet GY, Martin A, Martin V. Fatigue and recovery after high-intensity exercise part I: neuromuscular fatigue. Int J Sports Med. 2004; 25: 450-456.

[29] Patla AE. Strategies for dynamic stability during adaptive human locomotion. IEEE Eng Med Biol Mag. 2003; 22: 48-52.

[30] Gobbi LTB, Silva JJ, Barbieri FA. Do men and women use similar adaptive locomotion to clear static and dynamic obstacle? Human Movement. 2011; 12: 75-80.

[31] Lockhart TE, Smith JL,Woldstad JC. Effects of aging on the biomechanics of slips and falls. Hum Factors. 2005; 47: 708729.

32] Brach JS, Berthold R, Craik R, VanSwearingen JM, Newman AB. Gait variability in community-dwelling older adults. J Am Geriatr Soc. 2001; 49: 1646-1650.

[33] Mbourou GA, Lajoie Y, Teasdale N. Step length variability at gait initiation in elderly fallers and non-fallers, and young adults. Gerontology. 2003; 49: 21-26.

34] Hof AL, van Bockel RM, Schoppen T, Postema K. Control of lateral balance in walking - experimental findings in normal subjects and above-knee amputees. Gait Posture. 2007; 25: 250-258.

[35] Orendurff MS, Segal AD, Klute GK, Berge JS, Rohr ES, Kadel NJ. The effect of walking speed on center of mass displacement. J Rehabil Res Dev. 2004; 41: 829-834.

[36] Lee HJ, Chou LS. Detection of gait instability using the center of mass and center of pressure inclination angles. Arch Phys Med Rehabil. 2006; 87: 569-575.

[37] Staszkiewicz R, Ruchlewicz T, Forczek W, Laska J. The impact of changes in gait speed and step frequency on the extent of the center of mass displacements. Acta Bioeng Biomech. 2010; 12: 13-20.

[38] Petrella JK, Kim JS, Tuggle SC, Hall SR, Bamman MM. Age differences in knee extension power, contractile velocity, and fatigability. J Appl Physiol. 2005; 98: 211-220.

[39] Taimela S, Kankaanpaa M, Luoto S. The effect of lumbar fatigue on the ability to sense a change in lumbar position. A controlled study. Spine. 1999; 24: 1322-1327. 